\title{
Actual concepts of digital PAC-spectroscopy
}

\author{
J. Röder • C. Herden · J. A. Gardner · K. D. Becker · \\ M. Uhrmacher • H. Hofsäss
}

Published online: 6 August 2008

(C) The Author(s) 2008

\begin{abstract}
In conventional perturbed angular correlation (PAC)-spectroscopy huge amounts of events are processed by fast electronics. Modern digital signal processing devices and the improvement in the computer technology in recent years allow today digital PAC-spectrometer setups capable to perform software-based data processing with all the benefits of storage, repeatable data analysis under different limits, and easy switching between different isotopes. In this paper we discuss experiences and concepts of a first realized digital PAC-spectrometer, which will be rebuild at ISOLDE/CERN/Geneva.
\end{abstract}

Keywords Perturbed angular correlation (PAC) •

Digital signal processing $($ DSP $) \cdot$ Digital spectrometer $\cdot$ Linux

J. Röder · C. Herden · K. D. Becker

Institute of Physical and Theoretical Chemistry,

Braunschweig University of Technology, Braunschweig, Germany

J. Röder

e-mail: j.roeder@tu-bs.de

C. Herden

e-mail: cherden@viewplus.de

K. D. Becker

e-mail:k-d.becker@tu-bs.de

\section{J. A. Gardner}

Oregon State University, Corvallis, OR, USA

e-mail: john.gardner@viewplus.com

M. Uhrmacher $(\bowtie) \cdot$ H. Hofsäss

II. Institute of Physics, University of Göttingen, Göttingen, Germany

e-mail:muhrmac@gwdg.de

H. Hofsäss

e-mail: hhofsae@uni-goettingen.de 


\section{Introduction}

In perturbed angular correlation (PAC)-spectroscopy huge amounts of events in the order of $10^{5} \mathrm{~Hz}$ have to be processed which in conventional spectrometers is achieved by fast electronics. Coincidences are found by electronic logic operations and allow significant data reduction. Despite these benefits, the setting up of a traditional spectrometer is quite complex and time consuming. As a practical consequence the use of PAC isotopes is usually limited to very few PAC isotopes.

Modern digital signal processing (DSP) devices, such as digitizer or analyzer cards, allow an early digitalization of the photo multiplier signals and to handle the data for the coincidence search on standard personal computers (PC) by software analysis. Nowadays PCs have reached the performance to process and store data of a PAC experiment and to process them in real-time as conventional spectrometers do. A new generation TDPAC spectrometer has already been designed possessing many novel features $[1,2]$. In a conventional PAC setup, the number of detectors cannot be increased without an enormous increase in the complexity of the apparatus. The new design, however, permits the expansion to any number of channels. Each channel is peer to the other one and there is no precedence of which one becomes start and stop. This existing new spectrometer will serve as prototype for a second generation digital PAC spectrometer at ISOLDE/Geneva, where a wide range of PAC isotopes can be produced. The easy switching to other PAC isotopes with the new spectrometer will open new possibilities in PAC-spectroscopy.

\section{Concepts of digital signal and data processing in PAC-spectroscopy}

A variety of DSP devices exist, which require different concepts for the construction of a spectrometer. Here we focuses on digitizer and analyzer cards, single and multi channel input. Digitizer cards digitize the analog input and store the waveforms in their memory. These waveforms have to be analyzed outside the digitizer card on a PC. Analyzer cards are programmable and can run a fast waveform analysis within the card. However, analyzer cards are more expensive and often only available with lower sampling rate than digitizer cards.

The sharp rise up of the exponential PMT (photo multiplier) pulse form can be used to determine the arrival time of a $\gamma$-pulse accurately. In the DP110 from ACQIRIS with $1 \mathrm{GS} / \mathrm{s}$ (Giga Samples/second) the internal clock precision is $80 \mathrm{ps}$. Time interpolation techniques depend on the rise up sampling points of 1 ns width and allow an improved time resolution of 300 ps between detectors.

In order to determine the coincidences, data have to be exchanged between different PAC-channels. The fastest and most elegant solution is using programmable multi channel analyzer cards with the start and stop logic selection mechanism programmed into Field Programmable Gate Array (FPGA). FPGA have higher performances than PCs processors as data processing is very close to the basic hardware layer. Disadvantages are the limitation of the PAC-channel setup depending on the number of channels of the card, the need of reprogramming when changing isotopes, and the loss of the raw data, which need to be discarded and are not available for later analysis. We also found, that most available multi channel analyzer and digitizer 
cards were not able to trigger and store each of their channels fully independently. In fact this reduces the use of a multi channel card to a single channel card for PAC-spectroscopy.

To circumvent these disadvantage, the coincidence check procedure can be performed outside the cards fully software based on the PC. This requires the transfer of huge amounts of data, produced within a PAC experiment, from the cards to the PC. Storing and accessing these data again needs accurate performance planning.

Multi channel cards produce in total more data per card, which can find a limitation in the performance of the Peripheral Component Interconnect bus (PCI-bus) speed of the PC and other PC components such as hard drive speeds and network speeds for data transfer between the PCs. In the spectrometer design these factors have to be taken into consideration especially when several data flows occur at the same time and get limited by the PCs PCI-bus speed. Therefore, assigning to one PC only one PAC-channel has performance advantages over a multi channel setup on one PC.

Clock synchronization between the independent working cards is a major issue for a good working instrument. If the cards do not have interconnections between themselves, the cards clocks can be fed with an external clock signal, giving the cards the same time base.

Storing raw time and energy data of each PAC-channel on one PC without selection of start and stop makes it possible to record the entire PAC-experiment independently from decay times of different isotopes and their decay energies. Therefore no calibration before the experiment is required. A software based coincidence analysis can be easily performed by setting energy windows, time criteria, and channel widths. It can be performed as often as required as well as under different aspects or in more complex decays with different start and stops. Also the number of PAC-channels is not limited, when data are stored without any start and stop routing information, which only have to be implemented in the coincidence check software.

Data exchange between the PCs and process control becomes a major issue, which requires highly reliable and scalable operating systems. We found that Unix/Linux systems provide the best choice coming up with many useful standard tools and protocols, and high reliability.

As a PAC experiment produces hundreds of gigabytes of data per day, data reduction techniques become important. Many data contain non-coincidence events which can be discarded. We developed a software based filtering, called Open Window-Mode (OW-Mode) which stores the pure possible coincidences without any energy window selection but sorted with start and stop routing information. Re-examination of the reduced data with different energy window settings run up to 50 times faster than the Standard Energy Selective-Mode (SES-Mode).

In more complex decays of PAC isotopes, the amount of unusable data can be much higher than the coincidence data and reduce the efficiency of the instrument. To improve the throughput, a hardware pre-trigger logic can be used before the input to the instrument only passing possible coincidences.

The software based coincidence check procedure requires sufficient computer power as data from one channel has to be compared against all others. This scales with $2^{n}$ with $n$ as number of channel when zero-degree single time spectra are involved and with $2^{n}-n$ without them. As recorded data packets are sequenced, parallelization of the data can easily be performed running bundled sequences with 
different processes on different machines or in automatic load balancing clusters such as OpenMosix. [5]. This improves the performances especially for faster re-examination.

\section{The Braunschweig digital PAC-Spectrometer}

The basic concept of spectrometer developed in Braunschweig is a master-slave design. The master computer is the controlling unit for data acquisition and data analysis. The slave computers perform the data acquisition, equipped with one digitizer card, which digitizes the output of one photo-multiplier directly into waveforms. Within trigger limits, the signal of a detected photon is preselected from the background noise. the sharp rise up of the exponential waveform of the photon signal allows a precise determination of $\mathrm{t}_{0}$ using the time stamp of the trigger time of the digitizer card. Triggered waveforms are stored into the digitizer card memory. In a second step these data are transferred into the computers random access memory (RAM). The waveforms are then analyzed to determine photon energy by integration. Finally, energy and time stamp data are stored to the hard drive of each slave. The coincidence analysis is performed by the master computer, accessing the data stored on the slaves.

In Braunschweig, digitizer cards DP110 from ACQIRIs were used which store at maximum 8000 waveform segments in one measurement sequence before they have to be dumped from the cards memory to the computer and a new measurement sequence can start. The maximum recording speed is limited by dead times during write procedures within the digitizer card and by data transfer from the digitizer card to the computer.

Synchronization of the digitizer's clocks is achieved by an external $10 \mathrm{MHz}$ common clock signal fed into the digitizer's external clock input. The clock counters of the digitizers run at a random start value. In order to determine a time zero value for the time counters, an artificial signal pulse is sent to the photo multiplier input to all digitizer cards at the same time. The first pulse in a measurement sequence is therefore well known having time zero. In order to feed the artificial synchronization pulse into the same line where the photo multiplier is mounted, multiplexer boards are used to switch between photo multiplier line and pulse generator line. Figure 1 provides an overview of the setup. During a continuous measurement, the following communication steps are performed:

1. The master computer checks whether all slaves are ready for a measurement sequence.

2. The master computer switches all multiplexer boards to the pulse generator input.

3. The master computer release the pulse generator to send one pulse to all slaves.

4. The master computer switches the multiplexer boards back to photo multiplier line input.

5. The slave computers record events until the digitizer card's memory is full. Then the slave computer sends a signal to the master computer, which itself sends a stop recording signal to the remaining slaves computers. 


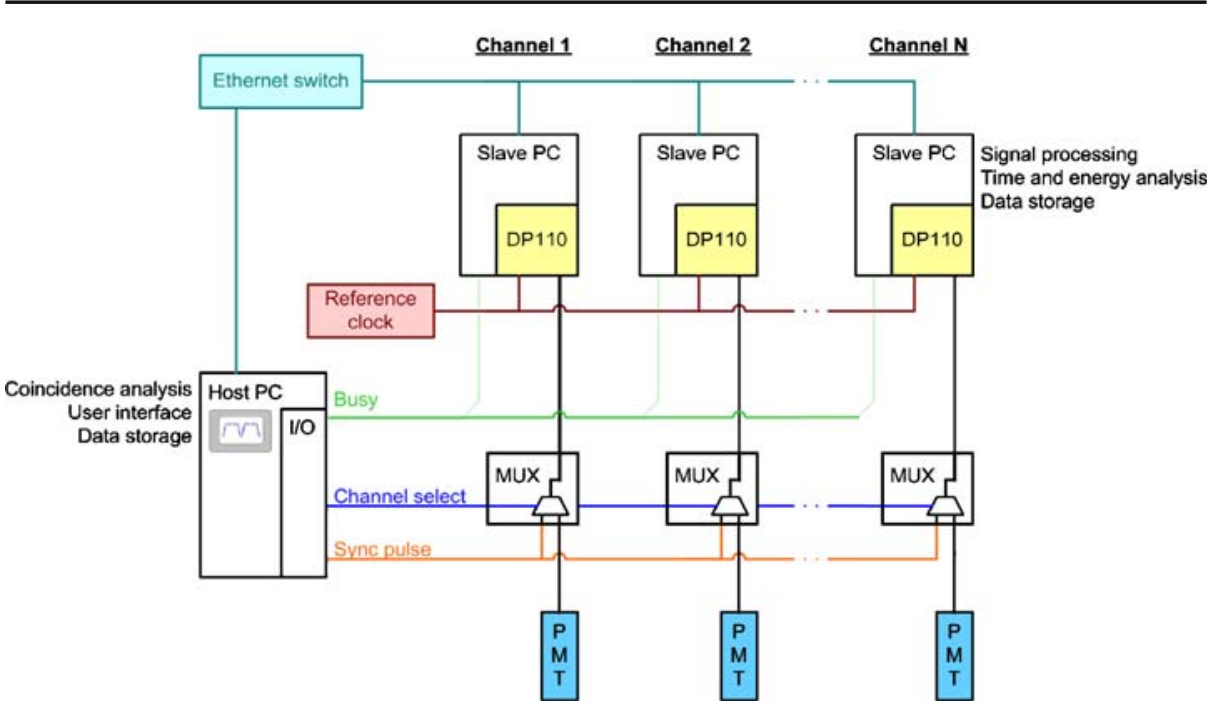

Fig. 1 Functional block diagram of the PAC setup. Due to the peer design of the PAC channels the apparatus can easily be expanded to any number of channels. The Busy (green), Channel select (blue), and Sync pulse (orange) signal lines are part of the handshake bus

Fig. 2 Raw data as an array of segments. Each segment having 400 data points.

The width of the data points depends of the defined sampling rate, which is here at $1 \mathrm{GS} / \mathrm{s}$. One segment contains the waveform of one event
Raw Data

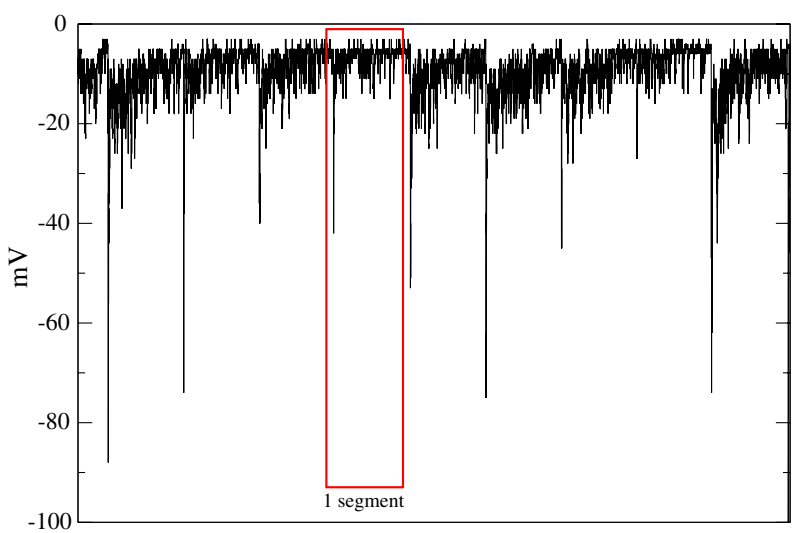

6. All slave computers write out the data from their digitizer cards to their RAM and signal the master computer to be ready for the next sequence, while the waveform analysis is performed as background process.

7. The procedure starts from the beginning.

Each slave computer records its data independently from the others within a sequence. The entire $\gamma$-events are recorded only as energy and time information, containing the start and stop events. Therefore there is no limitation for the number of channels in the PAC-spectrometer setup, as every slave is peer to the other. The 

Layers $(P L)$ of the digital PAC-spectrometer. Program names are indicated by double brackets: program()
Fig. 3 Software Program

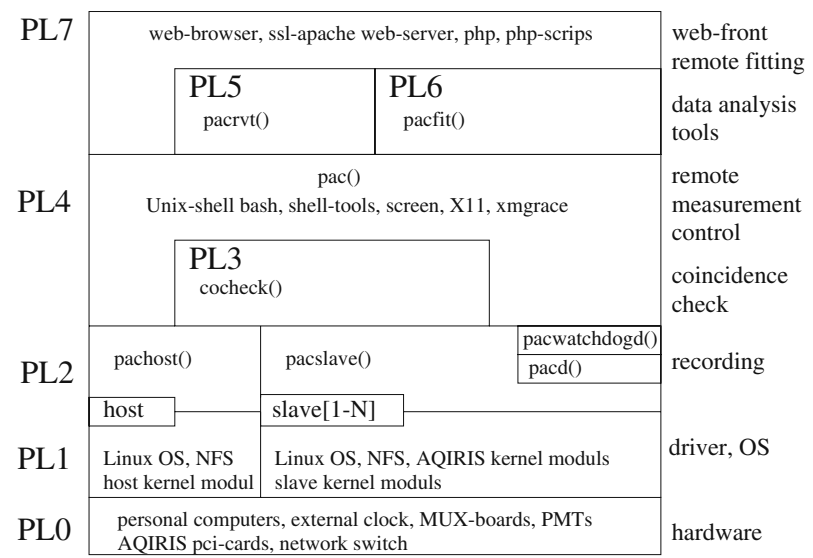

Table 1 Total coincidence rates for a four detector equipment at different activities

\begin{tabular}{lll}
\hline Activity $[\mathrm{MBq}]$ & OW-Mode $[\mathrm{kCo} / \mathrm{s}]$ & SES-Mode $[\mathrm{kCo} / \mathrm{s}]$ \\
\hline 0.7 & 13.9 & 2.0 \\
2.4 & 71.6 & 7.6 \\
8.4 & 260 & 26 \\
\hline
\end{tabular}

coincidence checking is a fully software based process, allowing easy setting of energy and time windows. It is run on the master computer accessing the data on the slaves. In this setup, there is no need of time calibration, because the clocks run with real time values. Changing between different isotopes is rather simple, as no calibrations are required. Only energy and time windows for the coincidence check have to be changed. Because all $\gamma$-events are stored to the hard drives of the slaves, the coincidence check can be performed as often as required, e.g. with different energy or time windows. Also only parts of the experiment can be investigated. Decays with more complex cascades permit to use different $\gamma$-energies for start and stop. In a standard measurement procedure where energy values are already known, the coincident check process can be started right after the recording process of the slaves, giving a real time snapshot of the actual measurement.

Figure 2 displays the raw data recorded by the digitizer card and saved as data array, with the box indicating one segment. These data remain only in the PCs RAM until integration and time analysis is finished. Figure 3 gives an overview of the software packets and their dependencies. The highly modularized software provides high flexibility in the data analysis, as many aspects can be easily controlled by stepwise investigation.

\section{Experimental results}

The actual equipment consists of the four slave PCs (Athlon XP 2800+ [2 GHz], 500 MByte RAM, 80 GByte hard drive space) and the host (Intel Pentium 4 [3.2 GHz] and 1 GByte RAM, 600 GByte hard drive space). A typical recording of $10^{5}$ sequences takes a total of about 60 GByte, (15 Gbyte per slave). Data accumulation rates dependent both on the rate of recording raw data and on the rate of computing 


\section{1}

In PAC - Fe-Foil PAC-BS
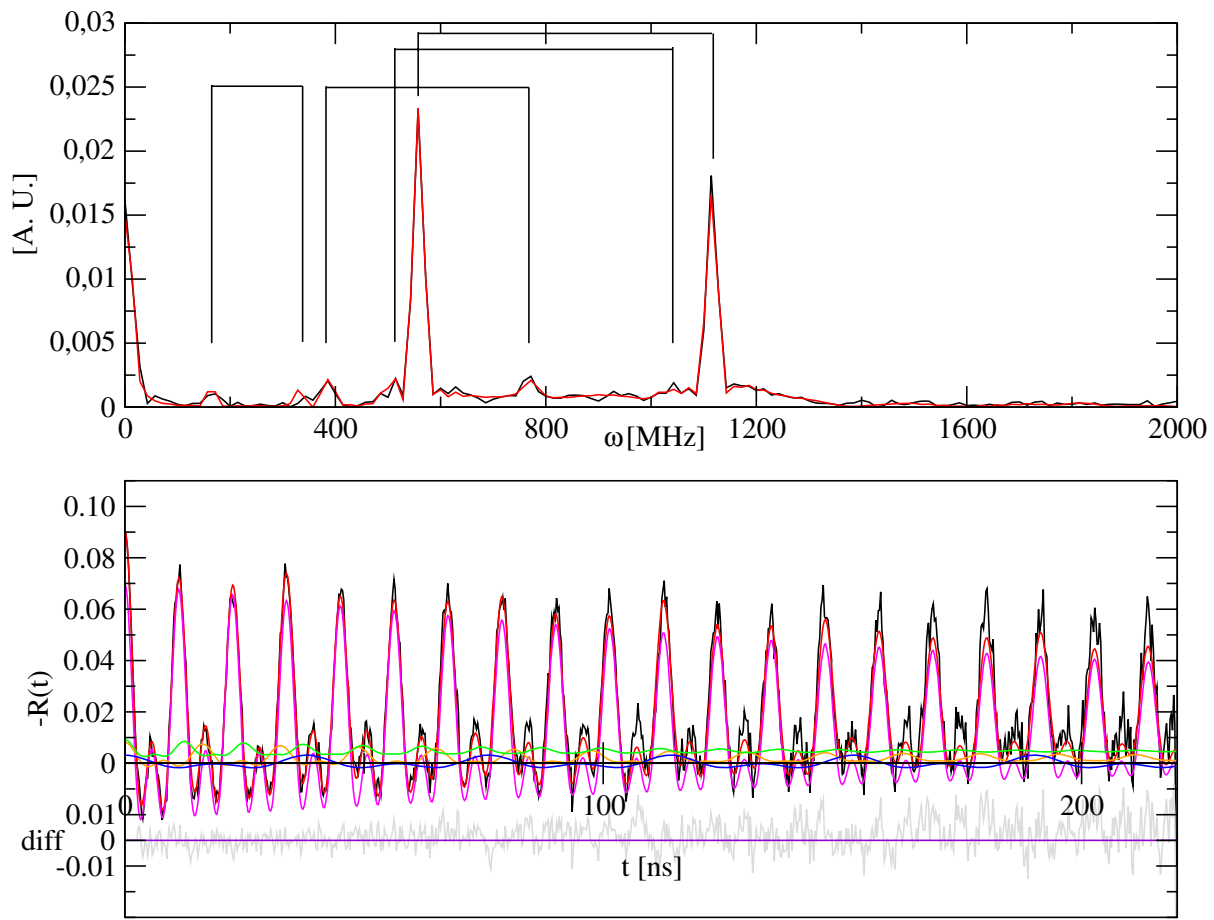

Fig. $4{ }^{111} \mathrm{In} /{ }^{111} \mathrm{Cd}$ PAC of an iron foil implanted with $400 \mathrm{keV}$ at IONAS/Göttingen. The foil was measured at room temperature as implanted without annealing

Table 2 Fit-data of Fe-foil

\begin{tabular}{llc}
\hline Fraction $[\%]$ & $\omega_{L}[\mathrm{MHz}]$ & $\delta[\mathrm{MHz}]$ \\
\hline 81.3 & $557(2)$ & 2.4 \\
8.6 & $505(66)$ & 11.1 \\
7.5 & $386(33)$ & 5.3 \\
2.6 & $165(56)$ & 0.0 \\
A22: & -0.12828 & \\
chi-squared: & 0.8378663 & \\
\hline
\end{tabular}

coincidence events. If raw data are being recorded sufficiently rapid, the cocheck program is mainly limited by computer speed. The bottleneck is the CPU (central processing unit) speed running the program. Data transfer between the slave and host computers is generally not a major factor. To process 60 GByte raw data by the cocheck program takes about $8.8 \mathrm{~h}$, which results in a process speed of $1.9 \mathrm{MByte} / \mathrm{s}$. Running in the OW-Mode the total coincidence rate, which the cocheck program can process as maximum speed, results in $35.9 \mathrm{kCo} / \mathrm{s}(\mathrm{kCo}=$ kilo coincidences per second) raw coincidences and in the SES-Mode in $5.5 \mathrm{kCo} / \mathrm{s}$ for a four detector setup.

A measurement finishes by a preset maximum sequence number, which is close to the number of coincidences. Thus, experiments can be conducted with well-defined 
and preset number of coincidences. For a sample activity of $0.7 \mathrm{MBq}$, which is calculated from the manufacturers calibration, the recording time for $10^{5}$ sequences is about $23 \mathrm{~h}$ and takes per sequence about 0.82 seconds. Each sequences includes a $120-150 \mathrm{~ms}$ fixed dead time for reading out the digitizer cards via the PCI-Bus, so that with higher sample activity the real recording time significantly decreases, but cannot become smaller than the dead time. For the prepared $0.7 \mathrm{MBq}$ sample at $70 \mathrm{~mm}$ distance of the PMTs which have $\mathrm{BaF}_{2}$ crystals of $25 \mathrm{~mm}$ diameter mounted, the activity was calculated from the data of a sequence data packet to be $0.4 \mathrm{MBq}$. This virtual amount indicates the efficiency of the $\gamma$-detection of the probe source, which is significantly smaller than the prepared source. Table 1 gives an overview of the obtained total coincidence rates for a four detector device giving 12 single spectra. The data are separated in OW-Mode and SES-Mode and depending on activity of the sample.

As a standard measurement to validate the accurate working of the instrument, an iron foil implanted with ${ }^{111}$ In was measured as implanted without annealing. Figure 4 and Table 2 illustrate the results with a strong signal at $558 \mathrm{MHz}$ which is identified as ${ }^{111} \mathrm{Cd}$ on a substitutional defect free iron site with $560 \mathrm{MHz}$ by [3, 6]. Whereas $\omega_{L}=505 \mathrm{MHz}$ may be assigned to a defect-associated site which is described in [4] to have $\omega_{L}=530(15) \mathrm{MHz}$. Additional frequencies have been found and can be assigned to beam-induced defects.

\section{Conclusions}

The fast development in electronics and computing of the recent years provides the basis for a change in PAC-spectroscopy from conventional fast electronic processing to a digital data processing analysis providing many new features and greater flexibility in the use of different PAC isotopes. The first successfully build digital PAC-spectrometer [1,2] will serve as prototype for a second spectrometer scaled up at ISOLDE/CERN/Geneva.

Acknowledgement Thanks are due to the DFG for founding in the framework of SPP 1136.

Open Access This article is distributed under the terms of the Creative Commons Attribution Noncommercial License which permits any noncommercial use, distribution, and reproduction in any medium, provided the original author(s) and source are credited.

\section{References}

1. Herden, C.H., Alves, M.A., Becker, K.D., Gardner, J.A.: A new generation TDPAC spectrometer. Hyperfine Interact. 159, 379 (2005)

2. Herden, C., Röder, J., Gardner, J.A., Becker, K.D.: Fully digital time differential perturbed angular correlation (TDPAC) spectrometer. Nucl. Instrum. Methods Phys. Res. A (2008). doi:10.1016/j.nima.2008.05.001

3. Lindgren, B., Vijay, Y.K.: Pressure and temperature dependence of the Cd-Hyperfine field in Fe, Co, Ni and Gd. Hyperfine Interact. 9, 379 (1981) 
4. Neubauer, M., Lieb, K.P., Uhrmacher, M., Wodniecki, P.: Thermal-spike effects in Xe-irradiated $\mathrm{Ag} / \mathrm{Fe}$ bilayers observed with PAC markers. Europhys. Lett. 43, 177 (1998)

5. Sloan, J.D.: High Performance Linux Clusters with OSCAR, Rocks, OpenMosix, and MPI. O'Reilly, Beijing (2004)

6. Wodniecki, P., Wodniecka, B., Kulinska, A., Lieb, K.P., Neubauer, M., Uhrmacher, M.: Indium solubility in iron studied with perturbed angular correlations. Hyperfine Interact. 120/121, 433 (1999) 